\title{
A Design and Implementation of Programming Examination Platform with Compilation Feature
}

\author{
Yifan Zhu1, a, Dali Yang1, band Jingwen Ma1, c \\ 1Computer School, Beijing Information Science and Technology \\ University, Beijing, China \\ azhuyf8899@mail.bistu.edu.cn, b yangdali@bistu.edu.cn,c \\ alexmajingwen@gmail.com
}

\begin{abstract}
.
With the popularization of education on computer science field and the development of courses reform, the amount of students who attend the class of Programming Technology is increasing. However, the demand of programming skill based on practice and the limited number of teacher determine that written examination cannot evaluate one's programming skill. Therefore, this article designed and implied a programming examination platform based on a LAMP (Linux, Apache, MySQL, PHP) server. With using Browser/Server method, the platform compiles code that provided by user and judged the correctness automatically. According to the experiment, the platform could evaluate one's programming skill more accurately.

Keywords: Computer Application Technology, Computer Network, Procedural Examination Platform.

\section{Section I: Introduction}

The examination platform is a method that examiners judge an examinee by evaluating his/her process in exam. In computer language study field, examination of process focuses on source code provided by examinees. Compared with the forms of traditional written exam, the platform with computers provides a better practice environment for examinees $\square$, which simulates an actual work environment. This exam method evaluates a paper by judging the answer generated from the source code which uploaded by examinees. It excludes the impact of examiners' subjective feelings that ensures the objectivity, timeliness and consistency of marking standards of the examination. It also enhances marking efficiency as well as quality. ${ }^{[1,2]}$

This article talks about a design of examination of process that it can compile $\mathrm{C}$ Language and $\mathrm{C}++$ Language questions and automatically run the source code provided by examinees and standard answer.
\end{abstract}


Since this article focuses on the process of program language examination, we define the programming examination platform discussed above as Procedural Examination Platform.

The rest of this paper is organized as follows. Functional design is given in Section II and key implement is given in Section III.

\section{Section II: Platform architecture}

Users of platform could be classified into three roles: administrator, teacher and student. The teachers and the students have a high similarity in competence in competence control because both of rules have the same purpose in using the platform. The administrators are an assistant role to handle the data generated by server and users. Three roles have different web directories to avoid errors caused by unauthorized access. ${ }^{[3]}$

Function structure. The students could edit their profiles, finish their homework uploaded by teachers, do exercises as well as challenges and attend exams. The teachers could edit their profiles, upload homework, manage students' list, manage the question list, manage course list and arrange exams. The Administrators could clean profiles of students and teachers, as well as reset record of exam, homework and question list. ${ }^{[4]}$

The function of homework could be described as Fig.1. A teacher uploads his homework requirements and actives the homework when everything is done. Then students would receive the homework notifications. After students have submitted their homework answer, the teacher will receive the answer sheet and give a remark.

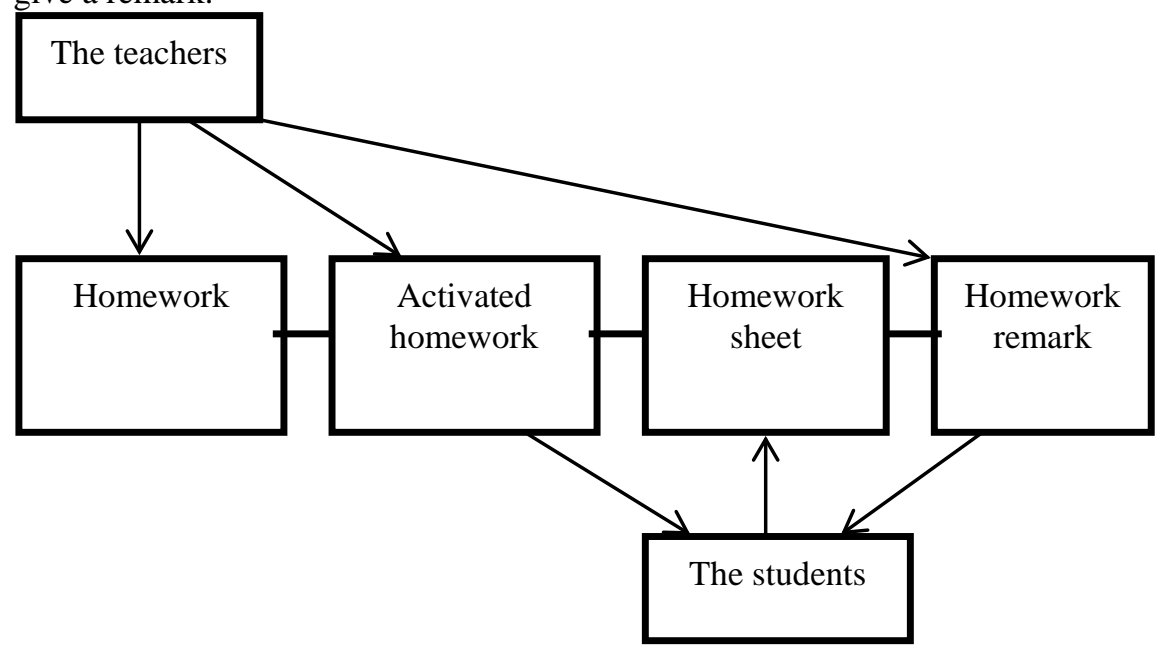

Fig. 1 Function of Homework

The function of exercises and exam is similar to homework function, the difference we gave to point out is that teachers could only manage questions in question list stored in database and students would take questions by random, the grades would also be evaluated by computers automatically. 
To import and export all students' information and exam result, the platform also provides an interface for teachers to import and export Excel files from database. In case of forgotten password, teachers could reset passwords of students.

Database design. Database would be used to store profiles, homework, exam, question lists and records. Therefore the design of database could be as Table 1 .

\begin{tabular}{|l|l|}
\hline Table name & Description \\
\hline admin & Profiles of administrators \\
\hline challenge_rules & Rule information in challenge model \\
\hline chapter & Information of chapters \\
\hline class & $\begin{array}{c}\text { Information of classes (lectures' } \\
\text { information included) }\end{array}$ \\
\hline exam_paper & Template of exam paper \\
\hline exam_paper_detail & Exam papers \\
\hline exam_place & Examination room information \\
\hline exam_place_detail & $\begin{array}{l}\text { IP address record in each examination } \\
\text { room }\end{array}$ \\
\hline knowledge & Knowledge point information \\
\hline plan & Exam plan \\
\hline stu_challenge & Records in students' challenge model \\
\hline stu_challenge_detail & $\begin{array}{c}\text { Code that students updated in challenge } \\
\text { model }\end{array}$ \\
\hline stu_homework & Homework which students uploaded \\
\hline stu_paper & Student's answer to paper \\
\hline stu_test & Recodes in students' test model \\
\hline stu_test_detail & Code that students updated in test model \\
\hline student & Information of students \\
\hline subject & Information of subjects \\
\hline te_homework & Homework template uploaded by teachers \\
\hline teacher & Information of teachers \\
\hline tiku & Question list \\
\hline tiku_bc_example & Test sample of Programming question \\
\hline
\end{tabular}

Table 1 Database design

Technical implementation. The application is implemented by using Codeigniter framework. Codeigniter uses a MVC (Model, View, Controller) model. In Codeigniter framework, controller handles the request from users; model processes data controlled by controller and return the data which controller demands and view responses the request controlled by controller. The workflow could be descripted as Fig. 2 . 


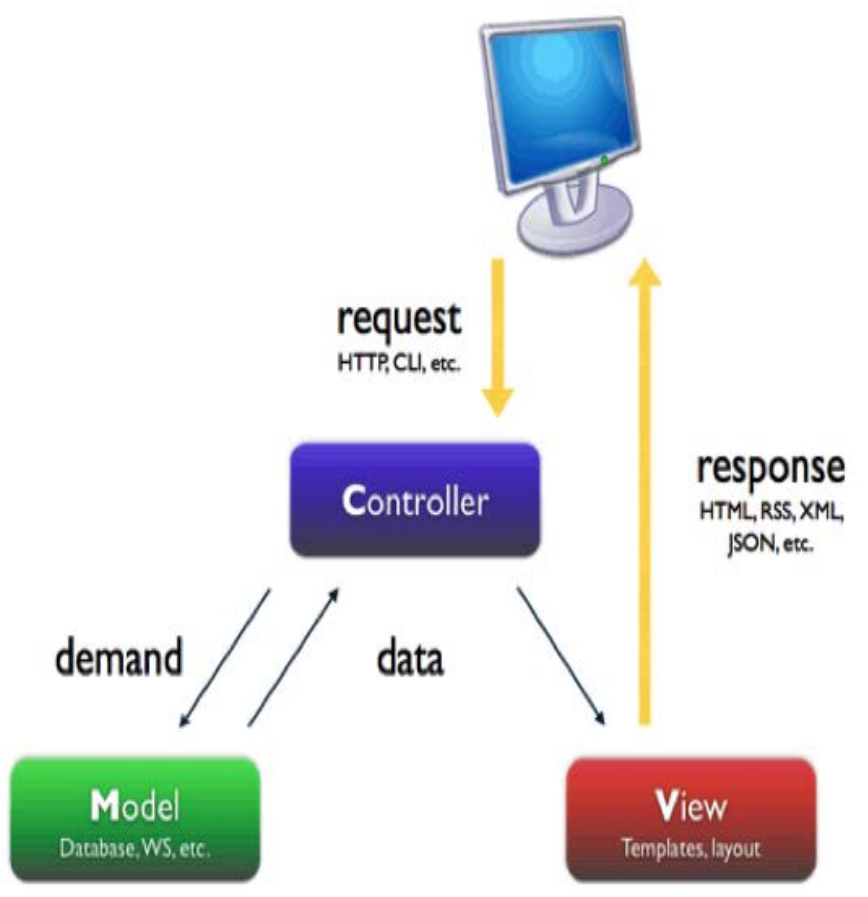

Fig. 2 MVC Model in Codeigniter

When students log in the platform, their IP address would be recorded to prevent students log in with same username. This situation would only be used in exam time. Besides, the exam paper would be generated automatically by exam paper template set by teachers so that students in adjacent seats would never use an exactly same paper (unless teachers only generate one sub-paper).

\section{Section III: Key technologies}

This section focuses on some key technologies to imply the platform. We mainly introduce two methods: way to make server compile code uploaded by students and asynchronous submission.

Code compilation. When a piece of code was uploaded to server, we firstly make a file in a specific directory whose owner is web server and directory name is user himself. Then the piece of code would be output to a new file then the web server use compiler (and interpreter) to run the code in a limit time and resource. ${ }^{[5]}$ At this moment, the web server would also redirect input test examples to the running program and record all the output generated by the program. After the limit of time the web server kills the process and stores the output into another file. The webserver would also compare the output file with 
standard output and evaluate grade by judge the trait of two files. The method could be described like Code.1: \$compiler is the type of compiler, \$codeText is the code that students upload, \$input is a string array that test example exists, \$filePath is the path that code would be stored, \$fileName is the name of code file, \$inname is the file name of input examples, \$outname is the file name where output stores and \$errorlog is file name for storing compile information.

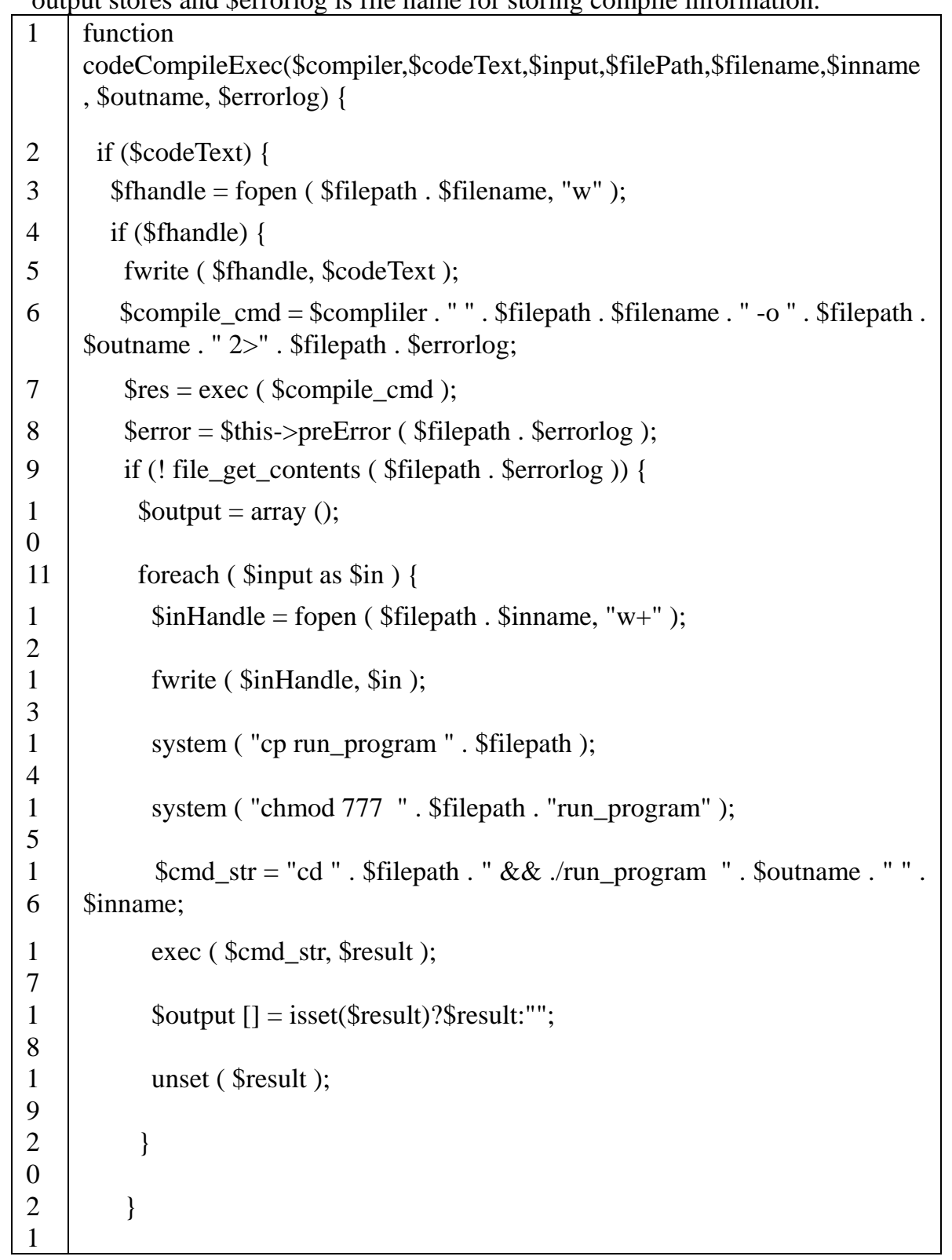




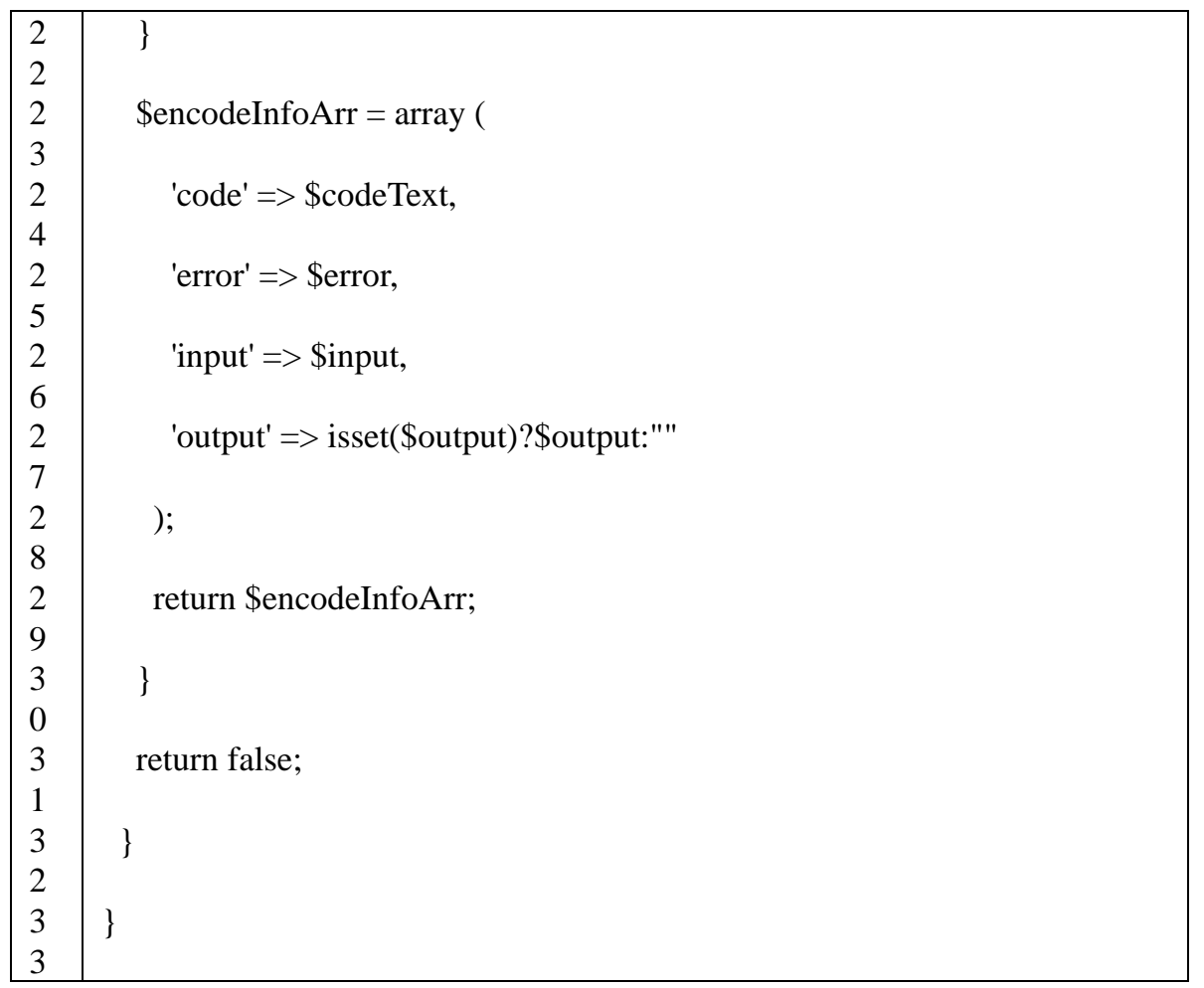

Code. 1 Code Compilation on Server

Asynchronous submission. If a student submitted a piece of code then he regarded, it is useless to click the "back" button because the browsers forbid backing to a submitted post form. Therefore the code submission and edit should be arranged at one page. Ajax (Asynchronous JavaScript And XML) provides a method to upload code asynchronously. Shown as Code.2, we use Ajax technology send the post form to another page, when that page responses the request, we can get the content the page provides and decide what would we do next. The input examples should be catenated by a special symbol (such like ' $<:$ : >') and the result should also be catenated as a long string which can be run by browser. ${ }^{[6]}$

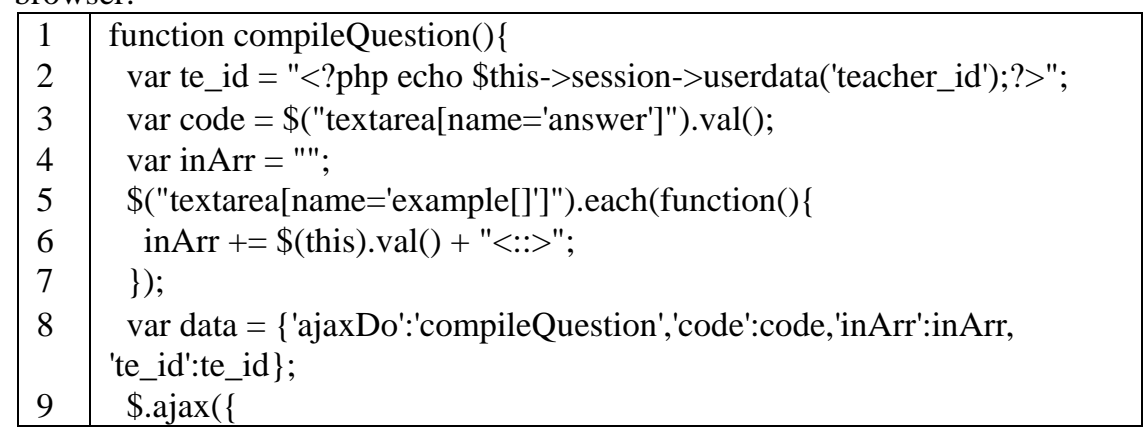




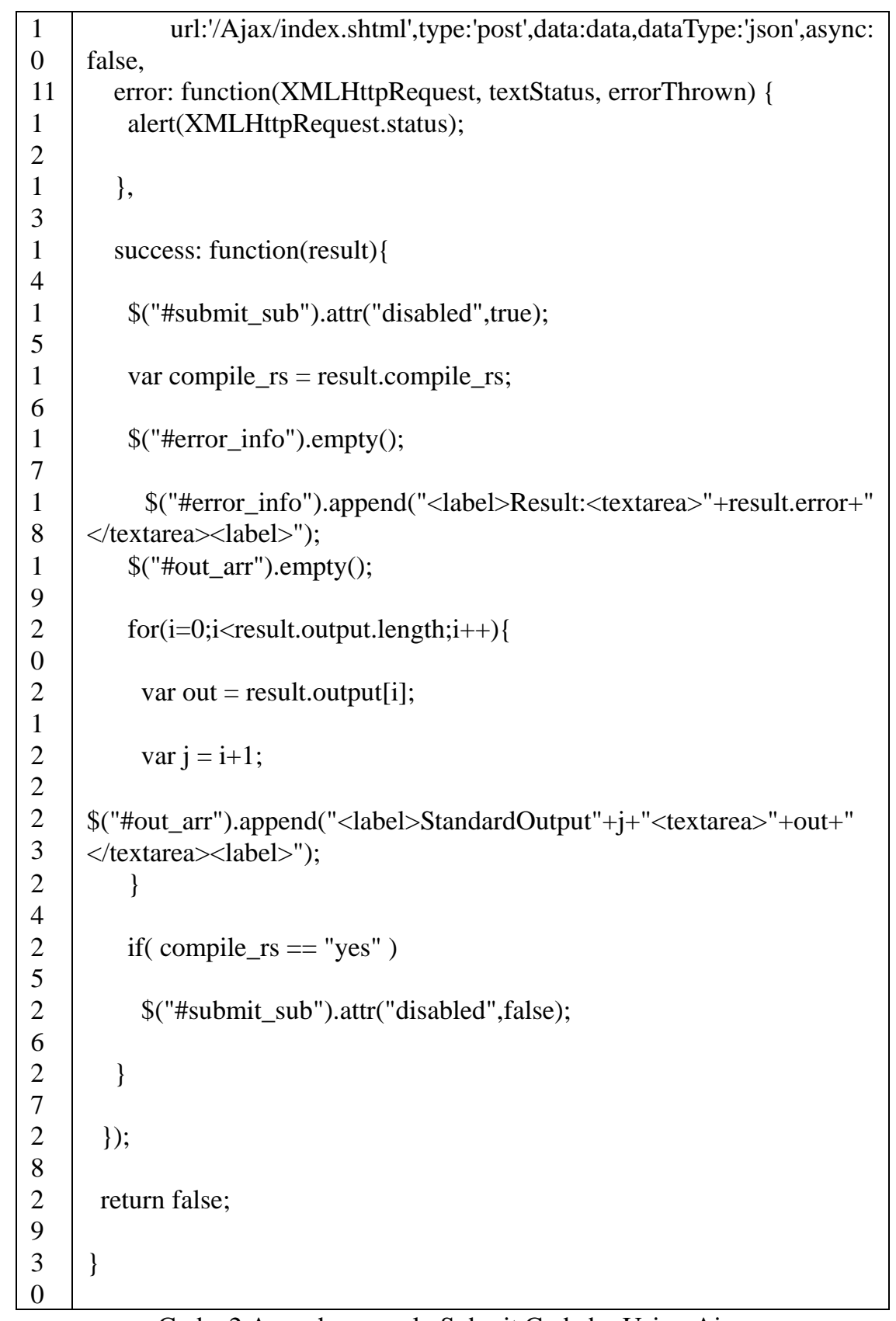

Code. 2 Asynchronous ly Submit Code by Using Ajax

\section{Section IV: Conclusions}


The platform mentioned in the article launched the Version 1.0 in 2013, which has been tested at Computer School of Beijing Information Science Technology University. According to the feedback of our test, this platform basically meets the requirements we discussed above.

During testing, Procedural test platform completes assignments like online training tasks and homework assigning. Besides, the challenge function is capable of selecting a group of programmers with superior capabilities. ${ }^{[7]}$

After a rewrite of the system, the performance and stability has been improved. We have launched Version 2.0 in March 2014. Procedural test platform completes the mission that we have mentioned previously: developing the coding ability of student, which is a requirement of our courses. Exam is an important way to examine the students' knowledge acquirement and to assess the effect of teaching. Therefore, it is an important indicator of teaching quality. The system greatly improves the efficiency of teachers while reduces the cost of the exams. With the development of network technology, it can be expected to become an indispensable examination choice, and gradually replace the traditional written examination. ${ }^{[8]}$

\section{Acknowledgements}

College Student Scientific Research Practice Program of Beijing Education Committee (PXM2014_014224_000079) financially supported this work.

\section{References}

[1] Dali Yang, Yifan Zhu and Jingwen Ma: Information Network Security Vol. 12 (2014), pp. 39-45, in Chinese.

[2] Zhikao Ren, Chen Ye and Guozu Liu: Information Processing (ISIP), 2010 Third International Symposium on.

[3] Yifan Zhu, Xing Huang and Hongzhe Liu: Computer CD Software and Applications Vol.16 (2014), pp.268-269, in Chinese.

[4] Keon Young Yi, Se Joong Jeon: Robotics and Automation, 1999. Proceedings. 1999 IEEE International Conference on.

[5] Yong Liu, Congfu Xu: Computer Applications and Software Vol.21, pp.21-22, In Chinese.

--- Wait end [6] Liangmiao Zhang, Xiaoping Zhang, Lang Liu, Xu Liu and Weiwei Rao: University Education, Vol.04 (2014), pp.89-90, in Chinese.

[7] Fu Yu and Zaiqiu Gu: China Management Informationization Vol. 12(2009), pp. 71-73, in Chinese. 
[8] Ming Zhou, Gang Qin, Yong Li: Journal of Chongqiong University Vol. 26(2003), pp.36-19, in Chinese. 